\title{
Efeito de pontas e pressões de pulverização na deriva de glyphosate + 2,4-D em condições de campo ${ }^{1}$
}

\author{
Effect of nozzles and pressures on spray drift of glyphosate $+2,4-\mathrm{D}$ in field \\ conditions
}

\begin{abstract}
Augusto Guerreiro Fontoura Costa $^{2}$, Edivaldo Domingues Velini ${ }^{3}$, Caio Vitagliano Santi Rossi ${ }^{4}$, Marcelo Rocha Corrêa ${ }^{5}$, Eduardo Negrisoli ${ }^{6}$, Marcus Vinicius Fiorini ${ }^{7}$, José Guilherme Ferreira Cordeiro $^{8}$, José Roberto Marques Silva ${ }^{9}$
\end{abstract}

Resumo - Objetivou-se com o trabalho avaliar o efeito de pontas e pressões de pulverização sobre a deriva de glyphosate $+2,4-\mathrm{D}$. Os modelos de pontas de pulverização e as respectivas pressões testadas foram: XR 11002 (150 e $300 \mathrm{kPa})$, DG 11002 (300 kPa), TT 11002 (300 kPa), AI $11002(300 \mathrm{kPa})$ e TXVS-10 (400 kPa). Para a coleta da deriva foram utilizados fios de náilon externos à área de aplicação $(1,5,10,20,50,100$ e $200 \mathrm{~m}$ de distância) com quatro repetições e seis cilindros de espuma posicionados sobre a barra do pulverizador. Sais traçadores foram adicionados a solução de aplicação para quantificar os depósitos de cada tratamento por meio de espectrofotômetro. Os herbicidas estiveram menos e mais suscetíveis a deriva com a ponta de pulverização de jato plano com pré-orifício e indução de ar (AI) e a de jato cônico vazio (TX), respectivamente. A seleção da ponta de pulverização e a redução na pressão de pulverização podem ser utilizadas para redução de deriva.

Palavras-chave: bico, pressão, herbicida, coletor de deriva, pulverizador de barra.

Abstract - The aim of the work was to evaluate the effect of nozzles and pressures on the spray drift from glyphosate $+2,4-\mathrm{D}$. The nozzles designs and the respective studied pressures were: XR $11002(150$ and $300 \mathrm{kPa})$, DG $11002(300 \mathrm{kPa})$, TT 11002 (300 kPa), AI 11002 (300 kPa) and TXVS-10 (400 kPa). Nylon strings outside the application area $(1,5,10,20,50,100$ and $200 \mathrm{~m}$ distance) with four replications and six foam cylinders placed on the boom of the sprayer were used to collect the spray drift. Salt tracers were added in the spray solutions to quantify the deposits of each treatment by spectrophotometer. Herbicides were less and more susceptible to

\footnotetext{
${ }^{1}$ Recebido para publicação em 10/11/2011e aceito em 11/02/2012.

${ }^{2}$ Pesquisador, Embrapa Algodão. R. Oswaldo Cruz, 1143, 58428-095, Campina Grande, PB. E-mail: augusto.costa@cnpa.embrapa.br

${ }^{3}$ Professor Dr., Depto de Produção Vegetal, FCA/UNESP, Núcleo de Pesquisas Avançadas em Matologia, Botucatu, SP.

${ }^{4}$ Engenheiro Agrônomo Dr., Milenia Agrociências S.A., Uberlândia, MG

${ }^{5}$ Engenheiro Agrônomo Dr., Techfield Assessoria e Consultoria Agrícola Ltda, Botucatu, SP.

${ }^{6}$ Engenheiro Agrônomo Dr., Techfield Assessoria e Consultoria Agrícola Ltda, Botucatu, SP; Professor, Faculdade de Agronomia EDUVALE, Avaré, SP.

${ }^{7}$ Engenheiro Agrônomo, Dow AgroSciences Industrial Ltda, São Paulo, SP

${ }^{8}$ Aluno de graduação da FATEC, Botucatu, SP.

${ }^{9}$ Aluno de graduação da USC, Bauru, SP
} 
Costa et al.

drift with the flat fan nozzle with pre-orifice and air induction (AI) and the hollow cone nozzle (TX), respectively. The nozzle selection and the reduction on the spray pressures may be used to decrease drift.

Keywords: tip, pressure, herbicide, drift collector, boom sprayer.

\section{Introdução}

A deriva, desvio de partículas ou gotas de tamanho reduzido formadas durante a pulverização que não atingem o alvo ou área alvo (Matuo, 1990; Miller, 1993), constitui uma das principais causas de perdas de herbicidas. A deriva torna-se indesejável principalmente pelos prejuízos diretos que ocasiona ao produtor como perdas financeiras com ações na justiça por danos a culturas sensíveis adjacentes; além de causar contaminação de alimentos, do ar e recursos de água e efeitos prejudiciais à saúde e segurança do ser humano, dos rebanhos bovinos e outras criações (Ozkan, 2011).

No sistema plantio direto há a necessidade de herbicidas para a dessecação no manejo de plantas daninhas, antecedendo o plantio das culturas, por não ser possível o revolvimento do solo. Dentre os herbicidas mais utilizados está o 2,4-D, com relevância pela reconhecida eficiência e relação custo benefício (Constantin \& Oliveira Jr., 2004), sendo comumente associado ao glyphosate para controle de um número maior de espécies. Apesar do conhecido efeito tóxico de subdoses de glyphosate que podem ser decorrentes da deriva (Yamashita \& Guimarães, 2006; Figueredo et al., 2007; Costa et al., 2009; Maciel et al., 2009; Gusmão et al., 2011), em geral, o 2,4-D representa maiores riscos de intoxicação às culturas fora da área alvo devido ao efeito em doses extremante baixas, as quais podem corresponder, por exemplo, a $1 \%$ do total aplicado quando atinge uma espécie sensível como o algodão (Constantin et al., 2007). Consequentemente, devido ao acentuado número de registros de danos causados às culturas em áreas vizinhas, o uso de 2,4-D tem sido restringido em diversos municípios brasileiros (Gazziero et al., 2000; Fagliari et al., 2004). Antuniassi (2006) também destaca o herbicida 2,4-D como um dos principais motivos para a preocupação com a deriva.

Christofoletti (1999) e Ozkan (2011) relataram que dentre as técnicas de aplicação, a seleção de pontas de pulverização representa uma das alternativas para redução de deriva, com o objetivo principal de aumentar o tamanho das gotas. Estudos em que foram utilizadas pontas de pulverização de baixa deriva ("low-drift") indicam a possibilidade de redução eficiente das perdas devido ao carregamento de agrotóxicos para fora das áreas-alvo (Lund, 2000; Cunha et al., 2003; Nuyttens et al., 2006; Schampheleire et al., 2006). Viana et al. (2010) relataram redução do risco de deriva devido ao maior tamanho de gotas obtido com a redução de pressão e seleção da ponta de pulverização. Resultados voltados para as aplicações de herbicidas, foram constatados com aplicações em préemergência a campo (Costa et al., 2007) e em condições controladas de túnel de vento com a mistura glyphosate + 2,4-D (Costa et al., 2008), sendo também observada diminuição do risco de deriva em função da diminuição da pressão de pulverização nos referidos estudos.

A partir da importância da seleção de tecnologias para a minimização dos riscos de deriva de herbicidas, objetivou-se com o trabalho avaliar o efeito de pontas e pressões de pulverização sobre a deriva de glyphosate + 2,4-D. 
Costa et al.

\section{Material e métodos}

O experimento foi realizado no município de Bocaina-SP, em área de pousio cedida pela Usina Santa Cândida (Tonon Bionergia S. A.). A área experimental foi de 27 ha, sendo a porção central de 1 ha $(50 \times 200 \mathrm{~m})$ utilizada para as aplicações e o restante para posicionar coletores de deriva. As plantas daninhas que compunham a cobertura vegetal possuíam cerca de $50 \mathrm{~cm}$ de altura, sendo as principais: Amaranthus spp., Brachiaria plantaginea, Brachiaria decumbens, Ipomoea grandifolia, Ipomoea quamoclit, Cyperus rotundus, Ricinus communis e rebrota de canade-açúcar (Saccharum spp.).

Os tratamentos foram constituídos por cinco condições operacionais de aplicação que corresponderam a modelos de pontas de pulverização combinadas a pressões de trabalho (Tabela 1). As pressões de pulverização foram utilizadas dentro dos respectivos intervalos recomendados pelo fabricante (Spraying Systems Co, 2004).

A velocidade do pulverizador foi de 4,3 $\mathrm{km} \mathrm{h}^{-1}$, sendo utilizados 4 bicos para cada tratamento (Tabela 1), espaçados a $50 \mathrm{~cm}$. Para que as aplicações de todos os tratamentos fossem simultâneas, foi utilizado um pulverizador pressurizado com $\mathrm{CO}_{2}$ desenvolvido especificamente para este tipo de estudo, com compartimento de calda, registros, válvulas reguladoras de pressão e seções de barra individualizadas. O mesmo foi acoplado a um pulverizador com barra de $11 \mathrm{~m}$ (Jacto PJ-400 - Figura 1A). As dosagens de herbicidas utilizadas correspondentes aos equivalentes ácidos foram de $1068 \mathrm{~g} \mathrm{ha}^{-1}$ de glyphosate e $670 \mathrm{~g} \mathrm{ha}^{-1}$ de 2,4-D, dos produtos comerciais Gliz 480 SL e DMA 806 BR, respectivamente. Para cada tratamento utilizou-se um sal traçador, sendo necessário preparar seis caldas de aplicação, as quais foram armazenadas em tanques de pulverização individuais com capacidade de $11 \mathrm{~L}$ acoplados ao trator (Figura 1A). Os sais utilizados foram $\mathrm{NaCl}, \mathrm{CaCl}, \mathrm{KCl}$, $\mathrm{MnSO}_{4}, \mathrm{MgCl}$ e $\mathrm{ZnCl}$, na concentração de $20 \mathrm{~g}$ $\mathrm{L}^{-1}$. Para cada aplicação foi retirada uma amostra de calda preparada para permitir posteriormente a quantificação exata do traçador em laboratório.

Tabela 1. Descrição dos tratamentos (pontas e pressões de pulverização).

\begin{tabular}{clc}
\hline Ponta de pulverização & \multicolumn{1}{c}{ Descrição } & Pressão (kPa) \\
\hline XR 11002 & Jato plano convencional de uso amplo & 150 \\
\hline XR 11002 & Jato plano convencional de uso amplo & 300 \\
\hline DG 11002 & Jato plano com pré-orifício & 300 \\
\hline TT 11002 & Jato plano defletor com pré-orifício & 300 \\
\hline AI 11002 & Jato plano com pré-orifício e indução de ar & 300 \\
\hline TX-10 & Jato cone vazio & 400 \\
\hline
\end{tabular}

Cilindros de espuma com $2400 \mathrm{~mm}$ de comprimento por $100 \mathrm{~mm}$ de espessura foram utilizados para coletar as gotas de menor tamanho que não atingissem diretamente o alvo, ficando suspensas na região da altura da barra de pulverização e, consequentemente, sob risco de deriva. Para tanto, seis unidades coletoras foram distribuídas e fixadas sobre a barra do pulverizador, com auxílio de armações de canos de PVC. Um cilindro foi posicionado acima de cada conjunto de pontas de pulverização, de modo a priorizar a coleta das gotas de cada tratamento (Figura 1A).

Os alvos utilizados como coletores de deriva foram fios de náilon com dimensão de 2 
Costa et al.

mm de diâmetro e 1200 mm de comprimento, os quais foram posicionados verticalmente em hastes metálicas (Figura 1B) fixadas ao solo nas distâncias de 1, 5, 10, 20, 50, 100 e 200 m da área de aplicação, sendo cada repetição composta por uma linha de coletores perpendicular e centralizada em um dos lados da área, totalizando 4 repetições. Alvos cilíndricos de $2 \mathrm{~mm}$ de espessura estão entre os coletores passivos considerados para a coleta e avaliação do risco de deriva, com as características desejáveis de dimensão reduzida, área definida, fracionável e facilidade no manuseio (Miller, 1993), sendo frequentemente utilizado em trabalhos a campo ou em condições controladas (Lund 2000; Costa et al., 2005; Costa et al., 2006, Costa et al., 2008; Moreira Junior \& Antuniassi, 2010; Souza et al., 2011).
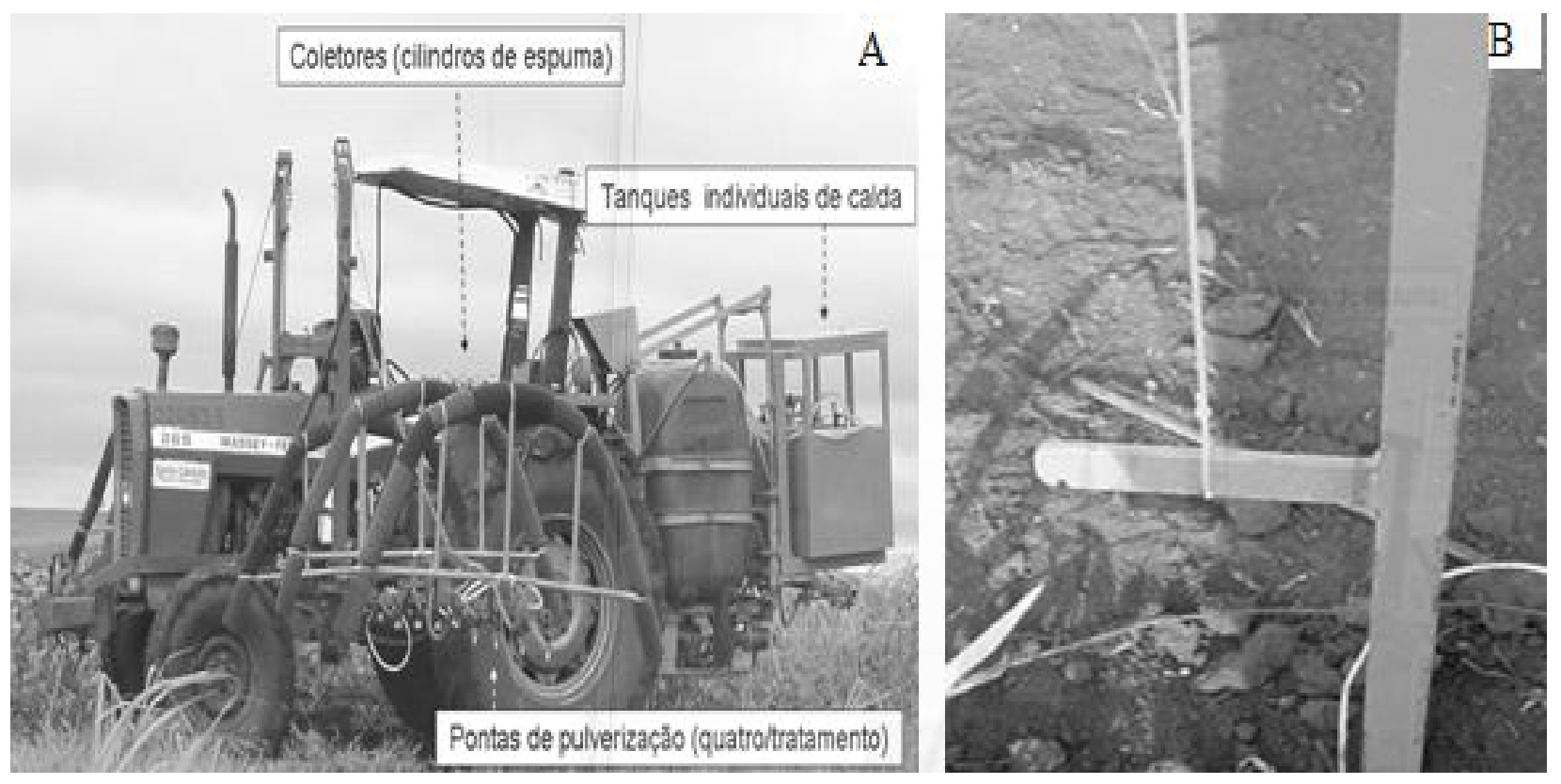

Figura 1. (A) Pulverizador utilizado para as aplicações, com destaque para o posicionamento dos tanques, pontas de pulverização e coletor (cilindro de espuma) acima da barra; (B) Detalhe do fio de náilon coletor de deriva fixado verticalmente em haste metálica em uma das distâncias de coleta externas a área de aplicação.

Foram realizadas dez aplicações em área total, a cada 50 minutos ao longo de um dia, iniciadas às $10 \mathrm{~h}$ e 05 min e finalizadas às $17 \mathrm{~h}$ e 36 min, permitindo que os dados obtidos fossem resultantes do somatório de depósitos em diferentes condições climáticas, além de favorecer a posterior detecção e quantificação dos traçadores. Durante e no intervalo entre as aplicações, a cada 30 segundos e 3 minutos, respectivamente, a velocidade do vento, temperatura e umidade relativa do ar foram registrados por meio de uma estação meteorológica portátil instalada na área experimental. As médias foram de $21,2 \mathrm{~km} \mathrm{~h}^{-1}$, $24,2{ }^{\circ} \mathrm{C}$ e $61,9 \%$, respectivamente. De acordo com as condições climáticas adequadas para aplicações de agrotóxicos por meio de pulverização, conforme critérios da ANDEF (2004), a velocidade se encontrava acima do limite de $10 \mathrm{~km} \mathrm{~h}^{-1}$. Portanto, favorável à comparação de tecnologias que pudessem 
Costa et al.

influenciar na magnitude do processo de deriva.

Ao final das aplicações, os fios de náilon e cilindros de espuma foram recolhidos e armazenados em frascos de PVC e sacos de polietileno, respectivamente. Posteriormente, em laboratório, cada uma das unidades coletoras foi lavada com água destilada com volumes conhecidos e as soluções de lavagem analisadas em espectrofotômetro de plasma. As concentrações dos traçadores foram corrigidas padronizando-se o volume de calda para $200 \mathrm{~L}$ $\mathrm{ha}^{-1}$ e transformadas em volume de calda depositado por unidade de área da superfície coletora $\left(\mu \mathrm{L} \quad \mathrm{cm}^{-2}\right)$. Para os coletores posicionados acima da barra de pulverização, a comparação entre os tratamentos foi realizada de maneira direta com as quantidades de depósitos obtidas. Para os alvos de fios de náilon as médias dos depósitos dos tratamentos foram comparadas utilizando-se os intervalos de confiança obtidos pela seguinte equação:

$$
\begin{gathered}
\frac{\mathrm{IC}=(\mathrm{t} x \text { desvpad }) / \text { raiz } \mathrm{nr}, \text { onde: }}{\mathrm{IC}=\text { intervalo de confiança; }} \\
\mathrm{t}=\text { valor de } \mathrm{t} \text { tabelado, ao nível de } 10 \% \mathrm{de} \\
\text { probabilidade; } \\
\text { desvpad = desvio padrão; } \\
\text { raiz } \mathrm{nr}=\text { raiz quadrada do número de repetições. }
\end{gathered}
$$

\section{Resultados e discussão}

A partir dos depósitos quantificados fora da área de aplicação (Figura 3), pode se observar quantidades decrescentes da calda principalmente até a distância de $20 \mathrm{~m}$. Resultados de campo encontrados na literatura também demonstram que quanto maior a distância de coleta, menor a deposição detectada fora da área alvo (Costa et al., 2005; Nuyttens et al., 2006). Em todas as distâncias de amostragem, não houve diferença estatística entre os tratamentos. Entretanto, considerandose as quantidades médias depositadas, principalmente até $20 \mathrm{~m}$ de distância, as pontas
TX e TT apresentaram os maiores valores de deriva, enquanto os demais tratamentos resultaram em menores quantidades detectadas.

Para os coletores posicionados acima da barra de pulverização (Figura 4), as quantidades observadas de depósitos foram maiores para a ponta de pulverização de jato cônico (TX). A ponta AI apresentou a menor quantidade coletada, seguida pela ponta XR na menor pressão e DG. Valores intermediários foram detectados para as pontas TT e XR a 300 $\mathrm{kPa}$. Considerando-se a menor quantidade de calda coletada para as pontas com indução de ar e/ou pré-orifício comparadas às convencionais, esses resultados são similares aos obtidos nos trabalhos de Lund (2000), Cunha et al. (2003), Nuyttens et al. (2006) e Schampheleire et al. (2006). Ao evidenciar o nível de diminuição de depósito fora da área alvo, Miller (2004) reportou que pontas com indução de ar reduziram a deriva entre 50 e $90 \%$, em relação a pontas convencionais. Fietsam et al. (2004) também verificaram que as aplicações com pontas de jato plano, envolvendo especificamente o herbicida glyphosate, demonstraram menor deriva quando utilizadas pontas de pré-orifício (DG) e indução de ar (AI), comparado ao modelo convencional XR.

De maneira semelhante, principalmente aos dados obtidos com o coletor acima a barra de pulverização, a partir de estudos de deriva de glyphosate + 2,4-D em condições controladas em túnel de vento com velocidade constante de 7,2 $\mathrm{km} \mathrm{h}^{-1}$, Costa et al. (2008) verificaram maior deriva para a ponta TX e menor deriva para a ponta AI, seguida pela ponta DG, com as mesmas pressões de pulverização utilizadas no presente estudo. Em ensaio a campo, utilizando amostrador dinâmico acima da barra de pulverização em aplicações de pré-emergência, Costa et al (2007) também constataram menor deriva para ponta de jato plano com indução de ar e maior deriva para ponta de jato cônico. Corroborando 
Costa et al.

com as constatações do presente estudo, de recomenda-se a utilização de pontas de acordo com Viana et al. (2007), para reduzir a pulverização com indução de ar para aplicação deriva de gotas de herbicidas não seletivos, de herbicidas sistêmicos em pós-emergência.

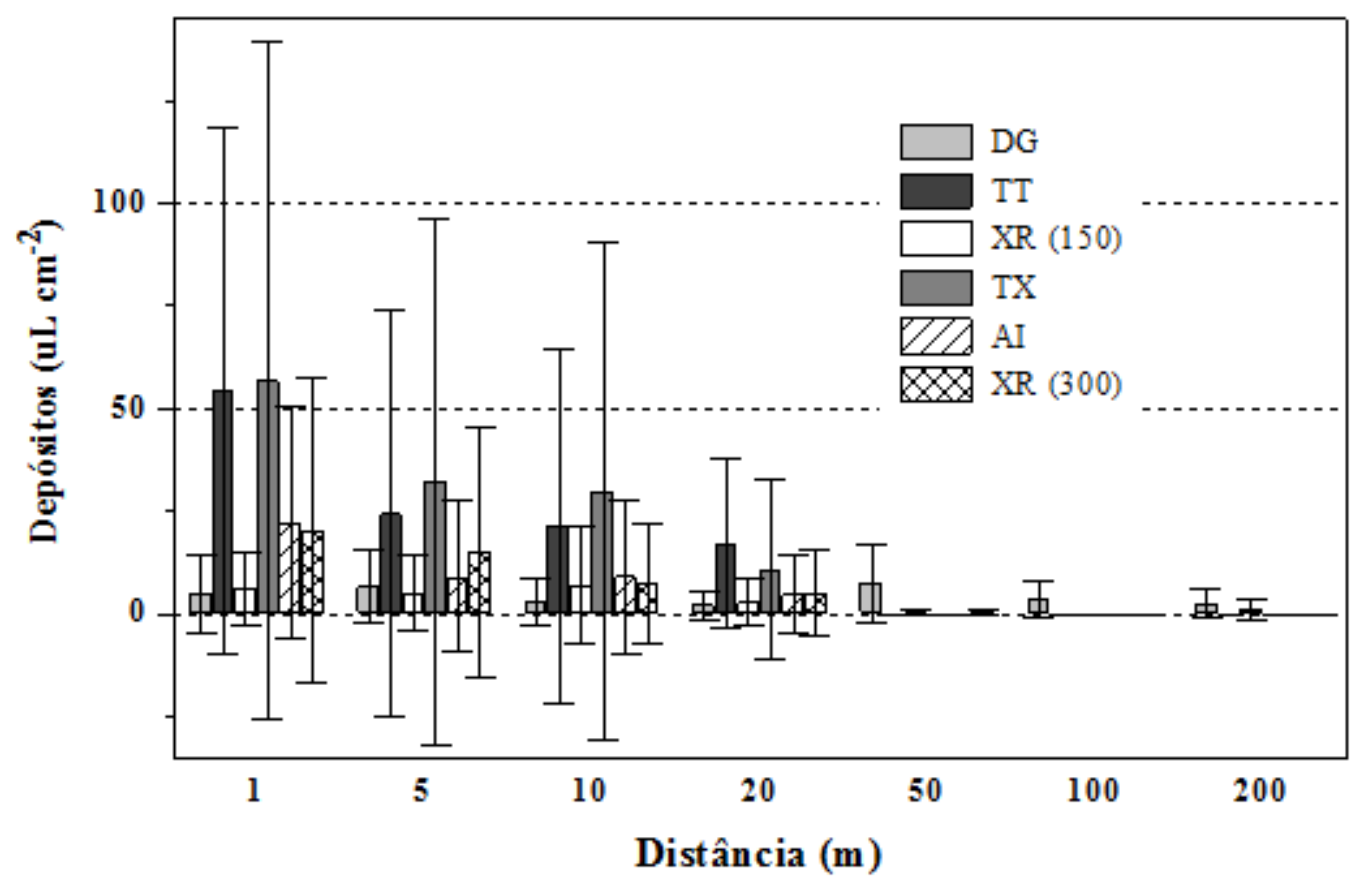

Figura 3. Depósitos de calda $\left(\mu \mathrm{L} \mathrm{cm}^{-2}\right)$ detectados nas posições de coleta fora da área de aplicação, em função das pontas de pulverização e pressões utilizadas.

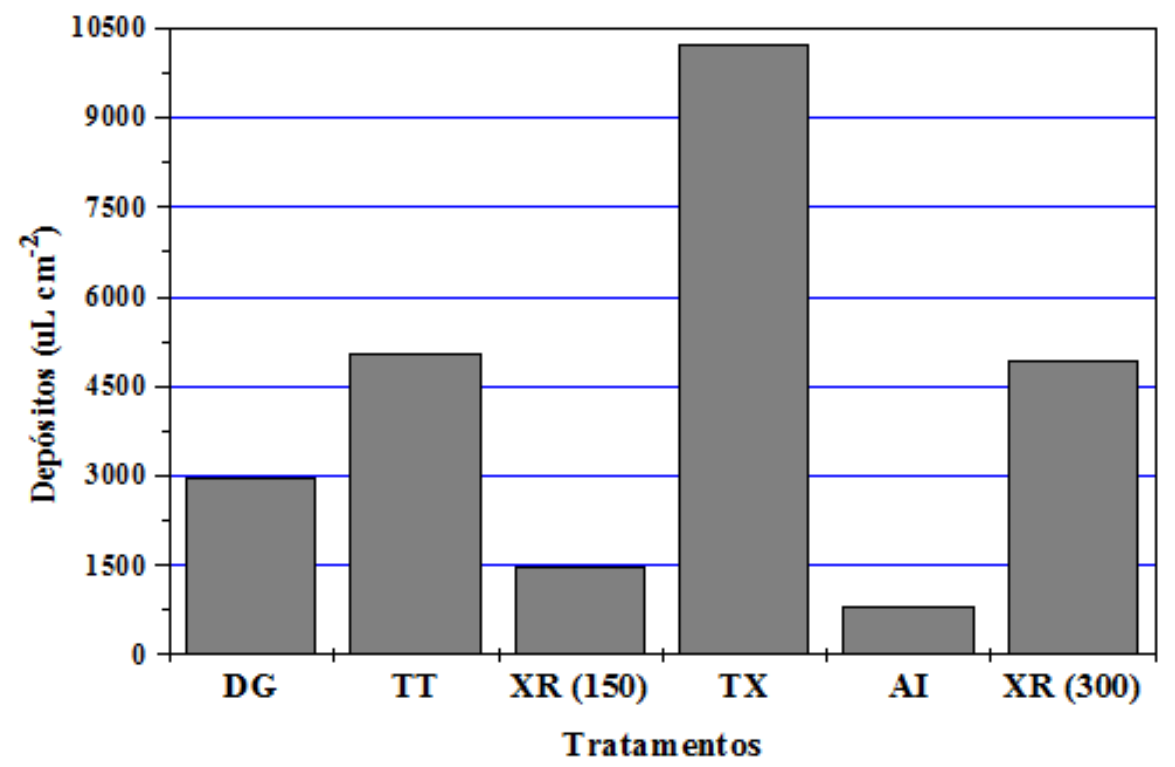

Figura 4. Depósitos de calda $\left(\mu \mathrm{L} \mathrm{cm}^{-2}\right)$ detectados nos coletores de espuma de cada tratamento, em função das pontas de pulverização e pressões utilizadas. 
$\mathrm{Na}$ deriva detectada até $10 \mathrm{~m}$ e nos depósitos quantificados acima da barra de pulverização (Figuras 3 e 4, respectivamente) observou-se menores valores na menor pressão utilizada para a ponta XR. Dinâmica semelhante foi constatada por Nuyttens et al. (2006) ao verificar redução de $35 \%$ na deriva, ao compararem uma aplicação com $190 \mathrm{kPa}$ em relação a outra com $300 \mathrm{kPa}$, utilizando a ponta de jato plano convencional F 11003. Para pontas de jato cônico, Costa et al. (2007), verificaram redução de $58 \%$ de deriva com a diminuição da pressão em aplicações de préemergência. Entretanto, a partir do estudo realizado, os autores consideraram a seleção da ponta de pulverização como estratégia potencialmente mais efetiva para diminuição da deriva de herbicidas.

Os menores valores de deriva e principalmente da quantidade de calda coletada acima da barra de pulverização podem ser explicados principalmente pelo maior tamanho das gotas pulverizadas. De acordo com Costa et al. (2008), a ponta AI apresentou o maior diâmetro mediano volumétrico (DMV) e a menor porcentagem do volume pulverizado com gotas menores que $100 \mu \mathrm{m}$. O DMV e essa porcentagem passaram a ser, respectivamente, maior e menor com a redução da pressão de pulverização para a ponta XR em pulverizações com a mistura glyphosate $+2,4$ D. Trabalhos de Lund (2000), Cunha (2003), Hoffmann \& Kirk (2005), corroboram com essa justificativa, pois a partir dos quais também foi possível constatar menor deriva com tecnologias que resultaram em pulverizações com gotas de maior tamanho. Nesse sentido, Viana et al. (2007) informaram que o conhecimento do espectro de gotas é imprescindível para seleção de pontas de pulverização em função do potencial de deriva. Souza et al. (2010) relacionaram as condições climáticas adequadas e o tamanho das gotas utilizadas (médias a muito grossas) como prováveis causas da não constatação de deriva em aplicações de dessecação com 2,4-D, após utilizarem utilizaram pontas de jato plano, jato plano defletor com indução de ar e jato plano duplo duplo de pré-orifício, independente da taxa de aplicação (80 ou $130 \mathrm{~L} \mathrm{ha}^{-1}$ ).

\section{Conclusões}

A menor e maior suscetibilidade a deriva ocorreram com as respectivas pontas de pulverização AI 11002 (300 kPa) e TX-10 (400 $\mathrm{kPa})$.

A seleção da ponta de pulverização e a redução na pressão de pulverização se mostraram como estratégias que podem ser utilizadas para redução de deriva.

\section{Agradecimentos}

À Dow AgroSciences pelo suporte financeiro. À Tonon Bionergia S. A. pela área cedida e todo o apoio do engenheiro agrônomo Edson José Negrisoli.

\section{Referências}

ANDEF - ASSOCIAÇÃO NACIONAL DE DEFESA VEGETAL. Manual de tecnologia de aplicação de produtos fitossanitários. Campinas: Linea Creativa, 2004. 50 p.

ANTUNIASSI, U.R. Spray drift: comparative perspectives between South America and Europe. Aspects of Applied Biology, v.77, p. 267-273, 2006.

CHRISTOFOLETTI, J.C. Considerações sobre a deriva nas pulverizações agrícolas e seu controle. São Paulo: Teejet South America, 1999. 15 p. (Boletim técnico, 4).

CONSTANTIN, J. et al. Efeito de subdoses de 2,4-D na produtividade do algodão e suscetibilidade da cultura em função de seu estádio de desenvolvimento. Engenharia Agrícola, v.27, n. esp., p. 24-29, 2007.

CONSTANTIN, J.; OLIVEIRA JR, R.S. Efeito do estádio de desenvolvimento de culturas na sensibilidade a subdoses do herbicida 2,4-D. In: RAETANO, C.G.; 
ANTUNIASSI, U.R. Qualidade em tecnolgia de aplicação. Fepaf, 2004. p.40-48.

COSTA, A.G.F. et al. Determinação da deriva de 2,4-D e glyphosate com diferentes pontas de pulverização e adjuvantes. In: CONGRESO de la ASOCIACIÓN LATINO-AMERICANA DE MALEZAS, 28., CONGRESSO BRASILEIRO DA CIÊNCIA DAS PLANTAS DANINHAS, 26, 2008, Ouro Preto. Anais... Sete Lagoas: SBCPD / Embrapa Milho e Sorgo, 2008. CDROM.

COSTA, A.G.F. et al. Efeito da intensidade do vento, da pressão e de pontas de pulverização na deriva de aplicações de herbicidas em préemergência. Planta Daninha, v.25, n.1, p.203210, 2007.

COSTA, A.G.F. et al. Methods to estimate losses in preemergence herbicide sprays. Bulletin of Environmental Contamination and Toxicology, v.74, n.1, p.8-15, 2005.

COSTA, A.G.F.; MILLER, P.C.H.; TUCK, C.R. The development of wind tunnel protocols for spray drift risk assessment. Aspects of Applied Biology, v.77, n.1, p.289294, 2006.

COSTA, N.V. et al. Efeito da deriva simulada de glyphosate no crescimento inicial de plantas de pinhão-manso. Planta Daninha, v.27, n.especial, p.1105-1110, 2009.

CUNHA, J.P.A.R. et al. Avaliação de estratégias para redução da deriva de agrotóxicos em pulverizações hidráulicas. Planta Daninha, v.21, n.2, p. 325-332, 2003.

FAGLIARI, J.R. et al. Efeitos e impacto econômico da aplicação de subdoses de 2,4-D, simulando deriva, sobre o tomateiro (Lycopersicon esculentum). Boletim Informativo Ciência das Plantas Daninhas, São Paulo, v.10, Suplemento, p. 200, 2004.

FIETSAM, J.F.W.; YOUNG, B.G.; STEFFEN, R.W. Herbicide drift reduction to drift control agents with glyphosate. Transactions of the ASAE, v.47, n.5, p.1405-1411, 2004.
FIGUEREDO, S.S. et al. Influência de doses reduzidas do glyphosate no tomateiro (Lycopersicom esculentum Mill.). Planta Daninha, v.25, n.3, p.849-857, 2007.

GAZZIERO, D.L.P. et al. Herbicide alternatives for 2,4-D in no-till cropping systems. In: INTERNATIONAL WEED SCIENCE CONGRESS, 3, 2000, Foz do Iguaçu-Brasil. Abstracts.... Corvallis: Weed Science Society, 2000. p.134.

GUSMÃO, G.A.; RONDON NETO, R.M.; YAMASHITA, O.M. Deriva simulada de glyphosate em plantas jovens de jenipapo. Revista Brasileira de Herbicidas, v.10, n.1, p.19-19, 2011.

HOFFMANN, W.C.; KIRK, I.W. Spray deposition and drift from two "medium" nozzles. Transactions of the ASAE, v.48, n.1, p. 5-11, 2005.

LUND, I. Nozzles for drift reduction. Aspects of Applied Biology, v.57, p.97-102, 2000.

MACIEL, C.D.G. et al. Crescimento do curauá branco sob efeito de subdoses de glyphosate. Revista Brasileira de Herbicidas, v.8, n.1, p.11-18, 2009.

MATUO, T. Técnicas de aplicação de defensivos agrícolas. Jaboticabal: Funep, 1990. $139 \mathrm{p}$.

MILLER, P.C.H. Reducing the risk of drift from boom sprayers. In: RAETANO, C.G.; ANTUNIASSI,U.R. Qualidade em tecnologia de aplicação. Botucatu: Fepaf, 2004. p.110124.

MILLER, P.C.H. Spray drift and its measurement. In: MATTHEWS, G.A.; HISLOP, E.C. Application technology for crop protection. CAB International, 1993. p.101122.

MOREIRA JUNIOR, O.; ANTUNIASSI, U.R. Construção e validação de um túnel de vento para ensaios da estimativa da deriva em 
pulverizações agrícolas. Revista Energia na Agricultura, v. 25, n. 3, p. 118-136, 2010.

NUYTTENS, D. et al. Experimental study of factors influencing the risk of drift from field sprayers, Part 2: Spray application technique. Aspects Applied Biology, v.77, n.2, p.331339, 2006.

OZKAN, H.E. Reducing the spray drift (Bulletin 816-00). Disponível em: <ohioline.osu.edu/b816/index.html>. Acesso em: 14 out. 2011.

SCHAMPHELEIRE, M. et al. The assessment of spray drift damage for grassland in Belgium. Aspects of Applied Biology, v.77,n.1, p.275280, 2006.

SOUZA, L.A.; CUNHA, J.P.A.R.; PAVANIN, L.A. Eficácia e perda do herbicida 2,4-D amina aplicado com diferentes volumes de calda e pontas de pulverização. Planta Daninha, v.29, n.2, p.1149-1156, 2011.

SPRAYING SYSTEMS CO. Mobile Systems Products - Catalog 49AM. Wheaton: Spraying Systems Company, 2004. 176 p.

VIANA, R.G. et al. Características técnicas de pontas de pulverização LA-1JC e SR-1. Planta Daninha, v.25, n.1, p.211-218, 2007.

VIANA, R.G. et al. Distribuição volumétrica e espectro de gotas de pontas de pulverização de baixa deriva. Planta Daninha, v.28, n.2, p.439-446, 2010.

YAMASHITA, O.M; GUIMARÃES, S.C. Deriva simulada de glyphosate em algodoeiro: efeito de dose, cultivar e estádio de desenvolvimento. Planta Daninha, v.24, n.4, p.821-826, 2006. 